\title{
MORREY SPACE
}

\author{
CRISTINA T. ZORKO
}

ABSTRACT. For $1 \leq p<\infty, \Omega$ an open and bounded subset of $R^{n}$, and a nonincreasing and nonnegative function $\varphi$ defined in $\left(0, \rho_{0}\right], \rho_{0}=\operatorname{diam} \Omega$, we introduce the space $\mathcal{M}_{\varphi, 0}^{p}(\Omega)$ of locally integrable functions satisfying

$$
\inf _{c \in C}\left\{\int_{B\left(x_{0}, \rho\right) \cap \Omega}|f(x)-c|^{p} d x\right\} \leq A\left|B\left(x_{0}, \rho\right)\right| \varphi^{p}(\rho)
$$

for every $x_{0} \in \Omega$ and $0<\rho \leq \rho_{0}$, where $\left|B\left(x_{0}, \rho\right)\right|$ denotes the volume of the ball centered in $x_{0}$ and radius $\rho$. The constant $A>0$ does not depend on $B\left(x_{0}, \rho\right)$.

(i) We list some results on the structure, regularity, and density properties of the space so defined.

(ii) $\mathcal{M}_{\varphi, 0}^{p}$ is represented as the dual of an atomic space.

Given an open and bounded subset $\Omega$ of $R^{n}$, let $\rho_{0}=\operatorname{diam} \Omega, 0 \leq \lambda \leq n$, $1 \leq p<\infty$. We denote by $L^{p, \lambda}(\Omega)$ the space introduced by Morrey of locally integrable functions $f(x)$ for which there is a constant $A=A(f)>0$, such that

$$
\int_{B\left(x_{0}, \rho\right) \cap \Omega}|f(x)|^{p} d x \leq A \rho^{\lambda}
$$

for every $x_{0} \in \Omega$, and $0<\rho \leq \rho_{0}$, where $B\left(x_{0}, \rho\right)=\left\{x \in R^{n} /\left|x-x_{0}\right|<\rho\right\}$.

More generally, given $k \geq 0$, we say that $f(x)$ belongs to $\mathcal{L}_{k}^{p, \lambda}(\Omega)$ if there is a constant $A>0$ such that for every $x_{0} \in \Omega$, and $0<\rho \leq \rho_{0}$ we have

$$
\inf _{P \in \mathcal{P}_{k}}\left\{\int_{B\left(x_{0}, \rho\right) \cap \Omega}|f(x)-P(x)|^{p} d x\right\} \leq A \rho^{\lambda},
$$

where $P_{k}$ is the class of polynomials of degree $\leq k$.

We observe that $L^{p, 0}(\Omega)$ describes $L^{p}(\Omega)$ and that $\mathcal{L}_{0}^{p, n}(\Omega)$ is a slightly more restricted version of $\mathrm{BMO}$, the space of functions of bounded mean oscillation.

More generally, we can replace the second member in (1) by $\left|B\left(x_{0}, \rho\right)\right| \varphi^{p}(\rho)$, where || denotes the Lebesgue measure and $\varphi$ is a function from $\left(0, \rho_{0}\right]$ into $[0, \infty)$. Let $M_{\varphi}^{p}(\Omega)$ be the space so defined. In the same way, using (2) we define the space $\mathcal{M}_{\varphi, k}^{p}(\Omega)$.

Condition (1) is obtained with $\varphi(t)=t^{(\lambda-n) / p}$. So the Morrey space is related to some nonincreasing function $\varphi(t)$ such that $\varphi(t) \rightarrow \infty$ when $t \rightarrow 0$. This is the kind of functions we will consider here.

Let us set

$$
\|f\|=\|f\|_{L^{p}(\Omega)}+\inf _{A \in C} A^{1 / p} .
$$

Received by the editors July 29, 1985.

1980 Mathematics Subject Classification (1985 Revision). Primary 43A15, 46E30; Secondary 26A33.

Key words and phrases. Mean oscillation, Morey space, density, duality. 
This is a norm in $\mathcal{M}_{\varphi, k}^{p}(\Omega)$ and in $M_{\varphi}^{p}(\Omega)$, so we get a Banach space.

Though $R^{n}$ is not bounded, in the same manner we can define $M_{\varphi}^{p}\left(R^{n}\right)$ and $\mathcal{M}_{\varphi, k}^{p}\left(R^{n}\right)$. In this case we use $M_{\varphi}^{p}$ and $\mathcal{M}_{\varphi, k}^{p}$.

We list some results on the structure, regularity, and density properties of the generalized Morrey space.

Proposition 1. Let $\Omega$ be an open and bounded subset of $R^{n}$. Suppose that there exists a constant $B>0$ such that

$$
\left|\Omega \cap B\left(x_{0}, \rho\right)\right| \geq B \varphi^{n} \quad \text { for all } x_{0} \in \Omega, \rho \leq \rho_{0} .
$$

Let $\varphi(t)$ satisfy the following conditions:

(i) There is $0<D<1$ such that $\varphi(2 t) \leq D \varphi(t)$ for $0<t \leq \rho_{0}$.

(ii) $\varphi(t)$ is nonincreasing and $t^{n} \varphi^{p}(t)$ is nondecreasing.

Then, $M_{\varphi}^{p}(\Omega)$ and $\mathcal{M}_{\varphi, k}^{p}(\Omega)$ describe the same space.

PROOF. This result has been proved by Campanato [2] when $\varphi(t)=t^{(\lambda-n) / p}$, $0<\lambda<n$. The same proof applies in the general situation with minor changes.

Condition $0<D<1$ imposed in (i) implies that $\varphi(t)$ cannot be a constant function. In fact, in that case (1) would mean that $f(x)$ belongs to $L^{\infty}(\Omega)$ which is not true for every function in BMO.

Functions in $M_{\varphi}^{p}(\Omega)$ can be trivially extended to $R^{n}$.

Proposition 2. We suppose that $t^{n} \varphi^{p}(t)$ is nondecreasing in $(0, \infty)$. Then, given $f(x) \in M_{\varphi}^{p}(\Omega)$, its extension $f^{*}(x)$ defined as zero outside $\Omega$ belongs to $M_{\varphi}^{p}$.

PROOF. It is simple. For $x_{0} \in R^{n}$ and $\rho>0$ it suffices to consider several cases: whether $x_{0}$ belongs to $\Omega$ or not and $\rho \leq \rho_{0}$ or not.

This result is also true for the space $\mathcal{M}_{\varphi, k}^{p}$ and includes the case $\varphi(t)=t^{(\lambda-n) / p}$ for $\lambda \geq 0$.

We can deduce that the definition of BMO as $\mathcal{L}_{0}^{p, n}(\Omega)$ is more restricted than the definition given in [3]. In fact, function $\log t$ belongs to $\mathrm{BMO}\left(R^{+}\right)$but its extension as zero for $t \leq 0$ does not belong to BMO.

From the estimate obtained by John and Nirenberg in [3] for the distribution function in BMO, we deduce that when $\lambda=n$ and $k=0$, condition (2) is satisfied independently of the exponent $p$ which we have used. This is no longer true for $0<\lambda<n$, because as was proved in [1], we can get functions in $M_{\varphi}^{p}(\Omega)$ with a distribution function arbitrarily large.

Functions in $M_{\varphi}^{p}(\Omega)$ cannot be approximated by functions in $C^{\infty}(\Omega)$, nor even by continuous functions. In fact, we find a simple example in $L^{p, \lambda}(\Omega), 0<\lambda<n$ : for $x_{0} \in \Omega$ and $\rho_{1} \leq \rho_{0}$ such that $B\left(x_{0}, \rho_{1}\right) \subset \Omega$, if we define

$$
f_{x_{0}}(x)=\left|x-x_{0}\right|^{(\lambda-n) / p}, \quad x \in \Omega
$$

then we obtain

$$
\|f-h\| \geq 2^{-p-1}\left|S^{n-1}\right|
$$

for any continuous function $h(x)$ in $\Omega$, where $S^{n-1}=\left\{x \in R^{n} /|x|=1\right\}$. To see this it suffices to find $0<\rho \leq \rho_{1}$ such that

$$
\int_{B\left(x_{0}, \rho\right)}\left|f_{x_{0}}(x)-h(x)\right|^{p} d x \geq 2^{-p-1}\left|S^{n-1}\right| \rho^{\lambda} .
$$


If $M=\sup _{x \in B\left(x_{0}, \rho_{1}\right)}|h(x)|^{p}$, then

$$
\begin{gathered}
\int_{B\left(x_{0}, \rho\right)}\left|f_{x_{0}}(x)-h(x)\right|^{p} d x \geq 2^{-p} \int_{B\left(x_{0}, \rho\right)}\left|f_{x_{0}}(x)\right|^{p} d x-\int_{B\left(x_{0}, \rho\right)}|h(x)|^{p} d x \\
\geq 2^{-p}\left|S^{n-1}\right| \rho^{\lambda}-M\left|S^{n-1}\right| \rho^{n}=\left|S^{n-1}\right| \rho^{\lambda}\left(2^{-p}-M \rho^{n-\lambda}\right) .
\end{gathered}
$$

Then it suffices to take $0<\rho \leq \rho_{1}$ such that $\left(2^{-p}-M \rho^{n-\lambda}\right) \geq 2^{-p-1}$.

The situation changes when we consider the following subset of $M_{\varphi}^{p}$ :

$$
\bar{M}_{\varphi}^{p}=\left\{f \in M_{\varphi}^{p} \text { such that }\|f(x-y)-f(x)\| \rightarrow 0 \text { when } y \rightarrow 0\right\} .
$$

We have

PROPOSITION 3. Let $\varphi(t)$ be nonincreasing such that $t^{n} \varphi^{p}(t)$ is nondecreasing. Let $\psi(x) \in C^{\infty}\left(R^{n}\right)$ be supported in $B(0,1), \int \psi(x) d x=1,0 \leq \psi(x) \leq 1$, and $\psi_{j}(x)=j^{n} \psi(j x)$. Then

(i) If $f(x) \in \bar{M}_{\varphi}^{p}, f * \psi_{j}(x) \rightarrow f(x)$ in the $M_{\varphi}^{p}$ norm as $j \rightarrow \infty$.

(ii) If $f(x)$ can be approximated by functions in $C_{0}^{1}$, then $f(x) \in \bar{M}_{\varphi}^{p}$.

ProOF. (i) for $\rho>0, \varepsilon>0$

$$
\begin{aligned}
& {\left[\int_{B\left(x_{0}, \rho\right)}\left|f * \psi_{j}(x)-f(x)\right|^{p} d x\right]^{1 / p}} \\
& \quad \leq\left[\int_{|y| \leq 1 / j} \psi_{j}(y) d y \int_{B\left(x_{0}, \rho\right)}|f(x-y)-f(x)|^{p} d x\right]^{1 / p} \\
& \leq \varepsilon\|f\| \rho^{n / p} \varphi(\rho) \quad \text { if } j \text { is large. }
\end{aligned}
$$

(ii) Let $\rho>0, x_{0} \in R^{n}, \varepsilon>0$. Let $g(x) \in C_{0}^{1}\left(R^{n}\right)$ such that $\|f-g\| \leq \varepsilon / 3$. Then

$(*)$

$$
\begin{gathered}
{\left[\int_{B\left(x_{0}, \rho\right)}|f(x-y)-f(x)|^{p} d x\right]^{1 / p} \leq\left[\int_{B\left(x_{0} \rho\right)}|f(x-y)-g(x-y)|^{p} d x\right]^{1 / p}} \\
+\left[\int_{B\left(x_{0}, \rho\right)}|g(x)-f(x)|^{p} d x\right]^{1 / p} \\
+\left[\int_{B\left(x_{0}, \rho\right)}|g(x-y)-g(x)|^{p} d x\right]^{1 / p} \\
\leq c 2 \varepsilon / 3 \rho^{n / p} \varphi(\rho)+\left[\int_{B\left(x_{0}, \rho\right)}|g(x-y)-g(x)|^{p} d x\right]^{1 / p} .
\end{gathered}
$$

Let $d>1$ such that $\operatorname{supp}(g) \subset B(0, d-1)$. If we take $|y|<1$, then

$$
\begin{aligned}
& \leq c 2 \varepsilon / 3 \rho^{n / p} \varphi(\rho)+|y|\|\nabla g\|_{\infty}|B(0, d)|^{1 / p} \\
& \leq c 2 \varepsilon / 3 \rho^{n / p} \varphi(\rho)+\frac{|y|}{\varphi(d)}\|\nabla g\|_{\infty} c^{\prime} d^{n / p} \varphi(d) .
\end{aligned}
$$


For $\rho \geq d$, since $t^{n} \varphi^{p}(t)$ is nondecreasing, it suffices to take

$$
|y| \leq \frac{\varphi(d) \varepsilon / 3}{\|\nabla g\|_{\infty} c^{\prime}} .
$$

If $\rho<d$, since $\varphi(t)$ is nonincreasing, we get

$$
\begin{aligned}
& \leq c 2 \varepsilon / 3 \rho^{n / p} \varphi(\rho)+|y|\|\nabla g\|_{\infty} \rho^{n / p} \\
& \leq c 2 \varepsilon / 3 \rho^{n / p} \varphi(\rho)+\frac{|y|}{\varphi(d)}\|\nabla g\|_{\infty} c^{\prime} \rho^{n / p} \varphi(\rho)
\end{aligned}
$$

as in the other case.

Proposition 3 is also true if we replace $R^{n}$ for any $\Omega \subset R^{n}$, open and bounded.

Description of $\mathcal{M}_{\varphi, 0}^{p}$ as a dual space. Fefferman and Stein have characterized BMO as the dual of the Hardy space $H^{1} \cdot \mathcal{M}_{\varphi, 0}^{p}$ can also be viewed as the dual of an "atomic" space.

For $1<p<\infty$, and $B\left(x_{0}, \rho\right)$, we define $A_{B\left(x_{0}, \rho\right)}^{p, \varphi}$ as the set of functions $a(x)$ such that

(i) $\operatorname{supp}(a) \subset B\left(x_{0}, \rho\right)$,

(ii) $\int a(x) d x=0$,

(iii) $\|a\|_{p} \leq 1 /\left|B\left(x_{0}, \rho\right)\right|^{1 / q} \varphi(\rho), 1 / p+1 / q=1$.

Let $A^{p, \varphi}=\bigcup_{B\left(x_{0}, \rho\right)} A_{B\left(x_{0}, \rho\right)}^{p, \varphi}$. We define $H^{p, \varphi}$ as the set of functions $f(x)$ such that $f(x)=\sum_{i \geq 0} \lambda_{i} a_{i}(x)$ in the sense of distributions, where $\lambda_{i} \in R, a_{i}(x) \in A^{p, \varphi}$, and $\sum_{i \geq 0}\left|\lambda_{i}\right|<\infty$.

We define a norm in $H^{p, \varphi}$

$$
\|f\|_{H^{p, \varphi}}=\inf \left(\sum_{i \geq 0}\left|\lambda_{i}\right|\right) .
$$

Infimum if taken over all atomic decompositions of $f$.

In this section we only consider real valued functions.

Proposition 4. Let $1<p<\infty, 1 / p+1 / q=1$. If $\varphi(t)$ is nonincreasing and $t^{n} \varphi^{q}(t)$ is nondecreasing, then $H^{p, \varphi}$ is a Banach space.

Proof. Clearly \|\|$_{H^{p, \varphi}}$ defines a norm in $H^{p, \varphi}$. We must only see that $H^{p, \varphi}$ is complete.

If $\left\{f_{n}\right\}$ is a Cauchy sequence, we can choose a subsequence $\left\{f_{n_{k}}\right\}$ such that $\left\|f_{n_{k}}-f_{n_{k-1}}\right\|_{H^{p, \varphi}} \leq 2^{-k}$. We define

$$
f=f_{n_{1}}+\sum_{k \geq 2}\left(f_{n_{k}}-f_{n_{k-1}}\right) .
$$

Let $f_{n_{k}}-f_{n_{k-1}}=\sum_{i \geq 0} \lambda_{i}^{k} a_{i}^{k}$ be the atomic decomposition such that

$$
\sum_{i \geq 0}\left|\lambda_{i}^{k}\right| \leq\left\|f_{n_{k}}-f_{n_{k-1}}\right\|_{H^{p, \varphi}}+2^{-k}
$$

Then $\sum_{k \geq 0} \sum_{i \geq 0}\left|\lambda_{i}^{k}\right|<\infty$ and we have a decomposition for $f$, except the series converges in the $H^{p, \varphi}$ norm. But this convergence also takes place in the sense of distributions. 
If $\psi$ is a testing function supported in $B\left(x_{1}, \rho_{1}\right)$ and $a$ is an atom, we have

$$
\left|\int a(x) \psi(x) d x\right| \leq\|a\|_{p}\|\psi\|_{q} \leq \frac{1}{|B(x, \rho)|^{1 / q} \varphi(\rho)}\left(\int_{B(x, \rho) \cap B\left(x_{1}, \rho_{1}\right)}|\psi(x)|^{q} d x\right)^{1 / q} .
$$

If $\rho \leq \rho_{1}$, then $\varphi(\rho) \geq \varphi\left(\rho_{1}\right)$ and

$$
\left|\int a(x) \psi(x) d x\right| \leq \frac{1}{|B(x, \rho)|^{1 / q} \varphi\left(\rho_{1}\right)}\|\psi\|_{\infty}|B(x, \rho)|^{1 / q}=\frac{\|\psi\|_{\infty}}{\varphi\left(\rho_{1}\right)} .
$$

If $\rho \geq \rho_{1}$, then $\rho_{1}^{n / q} \varphi\left(\rho_{1}\right) \leq \rho^{n / q} \varphi(\rho)$ and

$$
\left|\int a(x) \psi(x) d x\right| \leq \frac{1}{\left|B\left(x, \rho_{1}\right)\right|^{1 / q} \varphi\left(\rho_{1}\right)}\|\psi\|_{\infty}\left|B\left(x, \rho_{1}\right)\right|^{1 / q}=\frac{\|\psi\|_{\infty}}{\varphi\left(\rho_{1}\right)} .
$$

Then, the result follows for any $g(x) \in H^{p, \varphi}$.

Consequently, since $f_{n}$ converges to $f \in H^{p, \varphi}$ Proposition 4 is proved.

Finally, we have the following duality result.

Proposition 5. Let $\varphi(t) \geq 0,1<p<\infty$, and $1 / p+1 / q=1$. For any $L \in\left(H^{p, \varphi}\right)^{*}$ there exists $g \in \mathcal{M}_{\varphi, 0}^{q}$ such that if $h(x) \in H^{p, \varphi}$ we have

$$
L(h)=\int g(x) h(x) d x .
$$

Moreover, if $f \in \mathcal{M}_{\varphi, 0}^{q}$ and $h \in H^{p, \varphi}$, then $\int f(x) h(x) d x$ is an element of $\left(H^{p, \varphi}\right)^{*}$.

PROOF. The last statement is simple. For $f \in \mathcal{M}_{\varphi, 0}^{q}$ and $a$ an atom of $A_{B\left(x_{0}, \rho\right)}^{p, \varphi}$ we have

$$
\int a(x) f(x) d x=\int a(x)\left(f(x)-c\left(x_{0}, \rho\right)\right) d x
$$

where $c\left(x_{0}, \rho\right)$ is the constant for which the infimum in (2) is attained. Then

$$
\begin{aligned}
\int a(x) f(x) d x & \leq\|a\|_{p}\left(\int_{B\left(x_{0}, \rho\right)}\left|f(x)-c\left(x_{0}, \rho\right)\right|^{q} d x\right)^{1 / q} \\
& \leq \frac{1}{\left|B\left(x_{0}, \rho\right)\right|^{1 / q} \varphi(\rho)}\|f\|\left|B\left(x_{0}, \rho\right)\right|^{1 / q} \varphi(\rho)=\|f\| .
\end{aligned}
$$

For any $h \in H^{p, \varphi}$ the affirmation follows immediately.

To prove the other statement we see first of all that $\left(H^{p, \varphi}\right)^{*} \subset L_{\text {loc }}^{q}$.

Let $L \in\left(H^{p, \varphi}\right)^{*}$. Let $B_{k}$ be an increasing sequence of balls which cover $R^{n}$. Let $T_{k}$ be the restriction operator from $R^{n}$ to $B_{k}$. Then $L \circ T_{k}$ belongs to $\left(L_{0}^{p}\left(B_{k}\right)\right)^{*}$, where $L_{0}^{p}\left(B_{k}\right)$ denotes the subspace of $L^{p}\left(B_{k}\right)$ of functions having mean value zero. In fact, if $f \in L_{0}^{p}\left(B_{k}\right)$, then

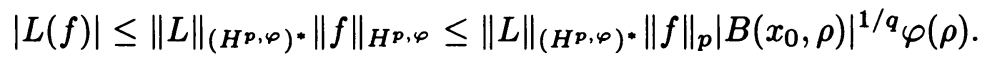

Since $\left(L_{0}^{p}\left(B_{k}\right)\right)^{*}=L^{q}\left(B_{k}\right) / C\left(B_{k}\right)\left(C\left(B_{k}\right)\right.$ is the space of the functions that are constant on $\left.B_{k}\right)$ there exists $g_{k} \in L^{q}\left(B_{k}\right)$ such that

$$
L(f)=\int f(x) g_{k}(x) d x .
$$


Since the $B_{k}$ are increasing we have $T_{k}\left(g_{k+1}\right)=g_{k}$. This implies the existence of a function $g \in L_{\text {loc }}^{q}$.

Now, we must prove that if $g \in L_{\text {loc }}^{q}$ is in $\left(H^{p, \varphi}\right)^{*}$, then $g$ belongs to $\mathcal{M}_{\varphi, 0}^{q}$. To see this, we use a constant $c\left(x_{0}, \rho\right)$ for which

$$
\begin{aligned}
& \left|\left\{x \in B\left(x_{0}, \rho\right) / g(x)<c\left(x_{0}, \rho\right)\right\}\right| \leq 1 / 2\left|B\left(x_{0}, \rho\right)\right|, \\
& \left|\left\{x \in B\left(x_{0}, \rho\right) / g(x)>c\left(x_{0}, \rho\right)\right\}\right| \geq 1 / 2\left|B\left(x_{0}, \rho\right)\right|,
\end{aligned}
$$

and suppose without loss of generality that

$$
\begin{aligned}
& \int_{B\left(x_{0}, \rho\right) \cap\left\{g(x)>c\left(x_{0}, \rho\right)\right\}}\left|g(x)-c\left(x_{0}, \rho\right)\right|^{q} d x \\
& \quad \geq \int_{B\left(x_{0}, \rho\right) \cap\left\{g(x) \leq c\left(x_{0}, \rho\right)\right\}}\left|g(x)-c\left(x_{0}, \rho\right)\right|^{q} d x .
\end{aligned}
$$

To simplify, we denote $A=B\left(x_{0}, \rho\right) \cap\left\{g(x)>c\left(x_{0}, \rho\right)\right\}$ and $B=B\left(x_{0}, \rho\right) \cap\{g(x) \leq$ $\left.c\left(x_{0}, \rho\right)\right\}$.

We define an atom $a(x)$ supported in $B\left(x_{0}, \rho\right)$ so

$$
a(x)=\left[g(x)-c\left(x_{0}, \rho\right)\right]^{q-1} \quad \text { for } x \text { in } A, \quad a(x)=C \quad \text { in } B\left(x_{0}, \rho\right) \backslash A,
$$

where $C$ is a constant chosen so that the mean value of $a(x)$ over $B\left(x_{0}, \rho\right)$ is zero.

We have

$$
\begin{aligned}
\int_{B\left(x_{0}, \rho\right)}\left|g(x)-c\left(x_{0}, \rho\right)\right|^{q} d x & \leq 2 \int_{A}\left|g(x)-c\left(x_{0}, \rho\right)\right|^{q} d x \\
& =2 \int_{A}\left(g(x)-c\left(x_{0}, \rho\right)\right) a(x) d x \\
& \leq 2 \int g(x) a(x) d x \leq 2\|g\|_{\left(H^{p, \varphi}\right) *\|a\|_{H^{p, \varphi}}}
\end{aligned}
$$

Now,

$$
\begin{aligned}
& \|a\|_{H^{p, \varphi}} \leq\|a\|_{p}\left|B\left(x_{0}, \rho\right)\right|^{1 / q} \varphi(\rho) \\
& \leq\left|B\left(x_{0}, \rho\right)\right| \varphi(\rho)\left[\frac{1}{\left|B\left(x_{0}, \rho\right)\right|} \int|a(x)|^{p} d x\right]^{1 / p} \\
& \leq\left|B\left(x_{0}, \rho\right)\right| \varphi(\rho)\left[\frac{1}{\left|B\left(x_{0}, \rho\right)\right|} \int_{A}\left|g(x)-c\left(x_{0}, \rho\right)\right|^{p(q-1)} d x+\frac{1}{\left|B\left(x_{0}, \rho\right)\right|} \int_{B} C^{p} d x\right]^{1 / p} .
\end{aligned}
$$

But

$$
\begin{aligned}
\frac{1}{\left|B\left(x_{0}, p\right)\right|} \int_{B} C^{p} d x & \leq\left[|B|^{-1} \int_{B} C d x\right]^{p} \\
& =\left[|B|^{-1} \int_{A}\left|g(x)-c\left(x_{0}, \rho\right)\right|^{q-1} d x\right]^{p} \\
& \leq|B|^{-1} \int_{A}\left|g(x)-c\left(x_{0}, \rho\right)\right|^{q} d x
\end{aligned}
$$

Hence

$$
\|a\|_{H^{p, \varphi}} \leq\left|B\left(x_{0}, \rho\right)\right| \varphi(\rho)\left[\frac{1}{\left|B\left(x_{0}, \rho\right)\right|} \int_{A}\left|g(x)-c\left(x_{0}, \rho\right)\right|^{q} d x\right]^{1 / p}
$$


Then

So, Proposition 5 is proved.

$$
\left[\int_{B\left(x_{0}, \rho\right)}\left|g(x)-c\left(x_{0}, \rho\right)\right|^{q} d x\right]^{1 / q} \leq c\left|B\left(x_{0}, \rho\right)\right|^{1 / q} \varphi(\rho) .
$$

\section{REFERENCES}

1. J. Alvarez Alonso, The distribution function in the Morrey space, Proc. Amer. Math. Soc. 83 (1981), 693-699.

2. S. Campanato, Proprietà di una famiglia di spazi funzionali, Ann. Sci. Norm. Sup. Pisa 18 (1964), 137-160.

3. F. John and L. Nirenberg, On functions of bounded mean oscillation, Comm. Pure Appl. Math. 14 (1964), 415-426.

4. J. L. Journé, Calderón-Zygmund operators, pseudo-differential operators and the Cauchy integral of Calderón, Springer-Verlag, 1983.

5. J. Peetre, On the theory of $L^{p, \lambda}$ spaces, J. Funct. Anal. 4 (1964), 71-87.

Casilla de Correo 244, (2300) Rafaela, Santa Fe, Argentina 